\title{
Depression, Stress, and Quality of Life in Persons with Chronic Kidney Disease: The Heart and Soul Study
}

\author{
Michelle C. Odden $^{a}$ Mary A. Whooleya, ${ }^{a}$ Michael G. Shlipak ${ }^{a, b}$ \\ ${ }^{\mathrm{a}}$ Section of General Internal Medicine, San Francisco VA Medical Center, and ${ }^{\mathrm{b}}$ Departments of Medicine and \\ Epidemiology and Biostatistics, University of California, San Francisco, Calif., USA
}

\section{Key Words}

Chronic kidney disease $\cdot$ Kidney disease, depression •

Kidney disease, stress $\cdot$ Kidney disease, quality of life

\begin{abstract}
Background: The effect of mild chronic kidney disease (CKD) on depression, stress, quality of life (QOL), and health status is not well understood. We compared these outcomes in subjects with and without CKD. Methods: We performed a cross-sectional study of 967 outpatients enrolled in the Heart and Soul Study. CKD was defined as a measured creatinine clearance $<60 \mathrm{ml} / \mathrm{min}$. Outcome measures included depressive symptoms measured using the Patient Health Questionnaire (PHQ), stress measured using the Perceived Stress Scale (PSS), and QOL and overall health rated as excellent, very good, good, fair, or poor. Results: The prevalence of depressive symptoms ( 17 vs. $19 \%, p=0.4$ ) or perceived stress ( 11 vs. $16 \%, p=0.09$ ) did not vary significantly by CKD. The prevalence of fair or poor QOL was not significantly different in subjects with CKD, compared with those without CKD (24 vs. $23 \%, p=0.65$ ). Age-adjusted analyses revealed a significant association of CKD with $\mathrm{QOL}(p=$ 0.003 ), however, this association no longer reached statistical significance after adjustment for confounders $(p=0.06)$. Subjects with CKD were more likely to report
\end{abstract}

poor or fair overall health than subjects without CKD (42 vs. $34 \%, p=0.03$ ). After multivariate adjustment, CKD remained significantly associated with worse overall health $(\mathrm{OR}=1.65,95 \% \mathrm{Cl} 1.21-2.24, \mathrm{p}=0.001)$, and modestly associated with $\mathrm{QOL}(\mathrm{OR}=1.31,95 \% \mathrm{Cl} 0.99-1.75$, $p=0.06)$, but had no association with depression ( $p=$ 0.48 ) or stress $(p=0.24)$. Conclusion: In this study of persons with coronary artery disease, subjects with CKD had reduced overall health and modestly reduced $\mathrm{QOL}$; however, mental health was similar in those with and without CKD. These findings suggest that selfassessed overall health may decline at earlier stages of renal dysfunction than mental health outcomes or QOL.

Copyright $\odot 2006$ S. Karger AG, Basel

\section{Introduction}

Advanced kidney disease is associated with poor quality of life (QOL), due to the effects of uremia on physical and mental health. End-stage renal disease (ESRD) leads to loss in mobility, exercise capacity, and self-assessed physical function, and is further associated with reduced ability to work and function in daily life [1-4]. In addition, many studies have found that a significant proportion of patients with ESRD suffer from depression [5-9].

\section{KARGER \\ Fax +4161306 1234 E-Mail karger@karger.ch} www.karger.com (c) 2006 S. Karger AG, Basel $1660-2110 / 06 / 1031-0001 \$ 23.50 / 0$

Accessible online at: www.karger.com/nec
Michael G. Shlipak, MD, MPH

San Francisco VA Medical Center, 4150 Clement St. (111A-1)

San Francisco, CA 94121 (USA)

Tel. +1 415221 4810/ext 3381, Fax +1 4153795573

E-Mailshlip@itsa.ucsf.edu 
The Modification of Diet in Renal Disease (MDRD) Study, a cohort of patients with moderate to severe chronic kidney disease (CKD), found decreased renal function to be associated with psychological distress and impaired health-related QOL [10].

A few studies have examined the physical component of QOL in subjects with mild to moderate renal dysfunction. In a prior analysis from the Heart and Soul Study, we found CKD was associated with self-assessed physical limitation [11]. Investigators from the Cardiovascular Health Study found elderly persons with CKD to have an increased prevalence of frailty [12]. In the African-American Study of Kidney Disease and Hypertension (AASK) Trial, the mean physical health score of the cohort was observed to be lower than in the general US population [13]. However, despite these recent findings, mental health outcomes and overall QOL in subjects with mild to moderate CKD have not been well characterized. Increasing interest in the association of ESRD with QOL has highlighted the relative paucity of similar evidence in subjects with CKD $[14,15]$.

The goal of this study was to determine whether moderate kidney disease is associated with reduced mental health outcomes and overall QOL in a population of ambulatory adults with chronic illness. Because QOL is a balance of health and psychosocial factors, we evaluated the relative effects of CKD in four different domains. Specifically, we compared depressive symptoms, perceived stress, QOL, and overall health, among participants with and without CKD in the Heart and Soul Study.

\section{Subjects and Methods}

\section{Subjects}

The Heart and Soul Study is a prospective cohort designed to examine the influence of psychosocial factors on cardiac outcomes in participants with known coronary disease. From September 2000 to December 2002, subjects were recruited from the San Francisco Veterans Affairs Medical Center, the Veterans Affairs Palo Alto Health Care System, the University of California, San Francisco Medical Center, and nine public health clinics in the Community Health Network of San Francisco. Subjects were eligible to participate if they had one of the following inclusion criteria: history of myocardial infarction, angiographic evidence of $>50 \%$ stenosis in one or more coronary vessels, evidence of exercise-induced ischemia by treadmill or nuclear testing, history of coronary revascularization, or documented diagnosis of coronary artery disease by an internist or cardiologist. Subjects were excluded if they had a myocardial infarction in the past 6 months, were unable to walk one block, or were planning to move out of the area in the next 3 years.
From 15,438 potential subjects, 1,024 were enrolled. Additional descriptions of the cohort have been previously published [16, 17]. For this analysis, we excluded subjects who did not have 24hour urine collection data $(n=57)$. All participants provided written informed consent, and the protocol was approved by the appropriate institutional review boards.

Measurements

Predictors

Primary. CKD was defined a priori as a creatinine clearance $<60 \mathrm{ml} / \mathrm{min}$ [18-20]. Creatinine clearance was measured, based on 24-hour urine collection, using the following formula: [Urine $\mathrm{Cr}$ $(\mathrm{mg} / \mathrm{dl}) \cdot 24$-hour urine volume $(\mathrm{ml})] /[$ Serum $\mathrm{Cr}(\mathrm{mg} / \mathrm{dl}) \cdot 1,440$ $(\mathrm{min} /$ day $)$. At the intake appointment, subjects were given a 3-liter collection jug and instructed to save all urine between the end of their intake appointment and the time when a researcher recovered the urine. Research personnel visited the subjects' homes exactly $24 \mathrm{~h}$ after their intake appointments to ensure accurately timed specimens. If the sample was reported to be incomplete, subjects were asked to repeat the collection, and research personnel returned $24 \mathrm{~h}$ later to re-collect the urine. Similarly, if the 3-liter collection jug was completely full, subjects were given two new jugs and asked to repeat the collection to insure that no urine was inadvertently discarded. If subjects were unable to collect all urine for any reason or had urinary incontinence, their samples were deemed inadequate and no urine creatinine data were recorded for these subjects.

Secondary. Age, sex, race, marital status, income, education, medical history, smoking status, and alcohol use were determined by self-report. We measured height and weight, and calculated body mass index (BMI). Serum samples were collected for measurement of hemoglobin, albumin, and creatinine.

\section{Outcomes}

Depressive symptoms were measured using the 9-item Patient Health Questionnaire (PHQ) [21]. We categorized patients with a score of 10 or higher as having depressive symptoms [22]. Stress was measured using the 4-item Perceived Stress Scale (PSS), and we considered patients to have stress if they scored 9 or greater on the 16-point scale [23]. As a measure of overall QOL, we asked patients, 'Compared with other people your age, how would you rate your overall quality of life?' [24, 25]. Subjects could respond with one of the following choices: excellent, very good, good, fair, or poor. We also asked patients, 'Compared with other people your age, how would you rate your overall health?' Subjects had the same choice of responses as for the overall QOL question.

Statistical Analyses

We evaluated baseline characteristics of subjects by the presence of kidney disease using a t test for continuous variables and $\chi^{2}$ statistic for dichotomous variables. We compared prevalence of depressive symptoms and stress by presence of CKD using a $\chi^{2}$ statistic. For the overall health and QOL questions, we compared the responses of excellent, very good, and good, fair, and poor using a $\chi^{2}$ statistic.

We used logistic regression to determine the association of CKD with depressive symptoms or stress. To determine the association of the CKD with overall health and QOL, we used ordered logit regression. This method was chosen as it allows the outcome to be modeled as an ordinal variable (excellent, very good, good, fair/ 
Table 1. Characteristics of participants with and without CKD (creatinine clearance $<60 \mathrm{ml} / \mathrm{min}$ )

\begin{tabular}{lccc}
\hline & CKD $(\%)$ & No CKD $(\%)$ & p value \\
& $n=236$ & $n=731$ & \\
\hline Demographic & & & \\
Age, years & $72 \pm 11$ & $65 \pm 10$ & $<0.001$ \\
Male & $200(85)$ & $603(83)$ & 0.45 \\
White & $150(64)$ & $437(60)$ & 0.31 \\
Married & $101(43)$ & $313(43)$ & 1.00 \\
Income USD $>20,000$ & $122(52)$ & $380(52)$ & 0.94 \\
High school graduate & $203(86)$ & $644(88)$ & 0.40 \\
Comorbidities and measurements & & & \\
Creatinine clearance, ml/min & $46 \pm 11$ & $92 \pm 22$ & $<0.001$ \\
Hemoglobin, g/l & $14.1 \pm 1.3$ & $13.3 \pm 1.5$ & $<0.001$ \\
Hypertension & $184(78)$ & $497(68)$ & 0.003 \\
Diabetes mellitus & $71(30)$ & $174(24)$ & 0.06 \\
Heart failure & $57(24)$ & $108(15)$ & 0.001 \\
Angina & $144(61)$ & $441(60)$ & 0.81 \\
Albumin, g/l & $3.9 \pm 0.3$ & $3.8 \pm 0.4$ & $<0.001$ \\
Smoking, current & $48(20)$ & $139(19)$ & 0.67 \\
Alcohol, regular use & $59(25)$ & $216(30)$ & 0.18 \\
BMI, kg/m ${ }^{2}$ & $27 \pm 5$ & $29 \pm 5$ & $<0.001$ \\
Prior myocardial infarction & $147(62)$ & $372(51)$ & 0.003 \\
Prior stroke & $53(22)$ & $87(12)$ & $<0.001$ \\
Prior revascularization & $97(41)$ & $255(35)$ & 0.09 \\
\hline
\end{tabular}

poor), rather than a dichotomous outcome. We added the secondary predictors listed above into the ordered logistic and logit regression models to determine the independent association of CKD with each outcome. To further explore the association of kidney function with self-reported QOL and health status, we calculated age-adjusted and multivariate-adjusted mean creatinine clearance in each outcome category, based on the parameter estimates from linear regression models. Backward stepwise regression was used with a criterion of $p<0.10$ for inclusion. We tested for interactions between CKD and age and race, for predicting each outcome. All analyses were performed using Stata 8.0 (Stata Corp., College Station, Tex., USA).

\section{Results}

Of the 967 eligible Heart and Soul Study participants, 236 (24\%) had CKD. Compared with participants who had normal renal function, those with CKD were older, and were more likely to have anemia, hypertension, heart failure, hypoalbuminuria, and had a lower BMI (table 1). Subjects with CKD were also more likely to have a history of myocardial infarction, or stroke.

The 17\% (40/236) prevalence of depressive symptoms in subjects with CKD was similar to the 19\% (142/731) prevalence in subjects without CKD $(p=0.40)$. Perceived stress also did not significantly differ by renal function:
Table 2. Associations of $\mathrm{CKD}$ with worse overall health, worse QOL, depressive symptoms, and stress

\begin{tabular}{|c|c|c|}
\hline & \multicolumn{2}{|c|}{ CKD (creatinine clearance $<60 \mathrm{ml} / \mathrm{min}$ ) } \\
\hline & OR $(95 \% \mathrm{CI})^{\mathrm{a}}$ & $\mathrm{p}$ value \\
\hline \multicolumn{3}{|c|}{ Depressive symptoms ( $\geq 10$ on PHQ) } \\
\hline Age-adjusted & $1.10(0.73-1.64)$ & 0.65 \\
\hline Adjusted $^{\mathrm{b}}$ & $0.86(0.56-1.31)$ & 0.48 \\
\hline \multicolumn{3}{|c|}{ Stressed ( $\geq 9$ on PSS) } \\
\hline Age-adjusted & $0.86(0.54-1.37)$ & 0.53 \\
\hline Adjusted $^{\mathrm{b}}$ & $0.75(0.46-1.22)$ & 0.24 \\
\hline \multicolumn{3}{|l|}{ Worse QOL } \\
\hline Age-adjusted & $1.51(1.15-1.98)$ & 0.003 \\
\hline Adjusted $^{\mathrm{b}}$ & $1.31(0.99-1.75)$ & 0.06 \\
\hline \multicolumn{3}{|c|}{ Worse overall health } \\
\hline Age-adjusted & $1.95(1.47-2.58)$ & $<0.001$ \\
\hline Adjusted $^{\mathrm{b}}$ & $1.65(1.21-2.24)$ & 0.001 \\
\hline
\end{tabular}

${ }^{a}$ Calculated using logistic regression for depressive symptoms or stress and ordered logit regression for worsening QOL or overall health.

${ }^{\mathrm{b}}$ Adjusted for age, sex, race, marital status, income, education, anemia, hypertension, diabetes, heart failure, angina, prior MI, prior stroke, prior revascularization, hypoalbuminuria, smoking status, alcohol use, BMI ( $<$ 0.01). 
Table 3. Age-adjusted and adjusted mean levels of creatinine clearance by level of self-reported QOL or health status

\begin{tabular}{|c|c|c|c|c|}
\hline & \multicolumn{4}{|c|}{ Creatinine clearance, $\mathrm{ml} / \mathrm{min}$} \\
\hline & $\begin{array}{l}\text { age-adjusted mean } \\
(95 \% \mathrm{CI})\end{array}$ & $\mathrm{p}$ value & $\begin{array}{l}\text { adjusted }^{\mathrm{a}} \text { mean } \\
(95 \% \mathrm{CI})\end{array}$ & $\mathrm{p}$ value \\
\hline \multicolumn{5}{|l|}{ Quality of life } \\
\hline Poor/fair & $77.3(74.4-80.1)$ & 0.002 & $79.2(76.3-82.0)$ & 0.22 \\
\hline Good & $80.1(78.3-81.8)$ & & $80.3(78.6-82.0)$ & \\
\hline Very good & $82.9(80.8-84.9)$ & & $81.4(79.4-83.4)$ & \\
\hline Excellent & $85.6(82.3-89.0)$ & & $82.6(79.1-86.0)$ & \\
\hline \multicolumn{5}{|l|}{ Health status } \\
\hline Poor/fair & $77.2(74.7-79.6)$ & $<0.001$ & $78.7(76.3-81.1)$ & 0.03 \\
\hline Good & $80.9(79.3-82.6)$ & & $80.7(79.1-82.2)$ & \\
\hline Very good & $84.7(82.3-87.1)$ & & $82.6(80.2-85.0)$ & \\
\hline Excellent & $88.4(84.5-92.3)$ & & $84.6(80.6-88.5)$ & \\
\hline
\end{tabular}

a Adjusted for age, sex, race, marital status, income, education, anemia, hypertension, diabetes, heart failure, angina, prior MI, prior stroke, prior revascularization, hypoalbuminuria, smoking status, alcohol use, BMI ( $\mathrm{p}<0.01)$.

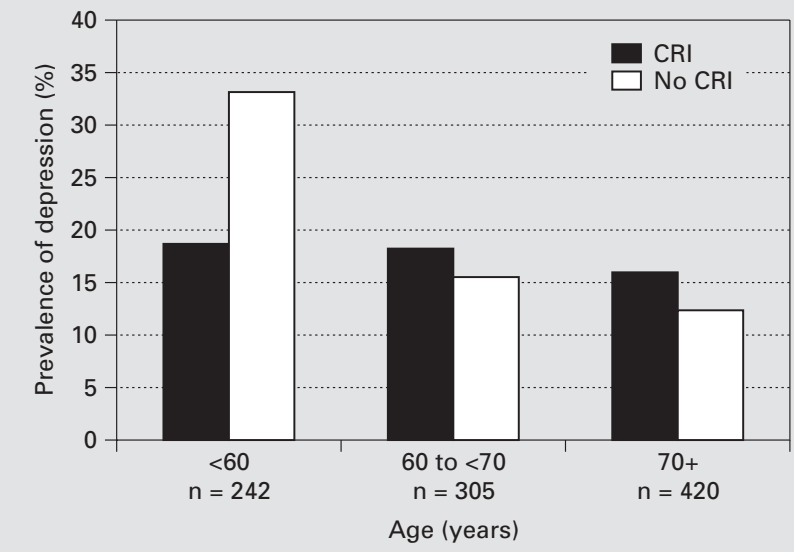

Fig. 1. Prevalence of depressive symptoms in subjects with and without CKD in subjects $<60,60$ to $<70$, and $70+$ years old.

27/239 (11\%) with CKD vs. $117 / 731$ (16\%) without CKD, $\mathrm{p}=0.09$. Using logistic regression, we found that CKD was not significantly associated with the mental health outcomes of depressive symptoms and stress in age-adjusted or adjusted analyses (table 2).

Of 236 subjects with CKD, 57 (24\%) reported fair or poor QOL vs. $166(23 \%)$ subjects without $\mathrm{CKD}(\mathrm{p}=0.65)$. $100(42 \%)$ subjects with CKD reported fair or poor overall health compared with 251 (34\%) with no CKD (p =
0.03). Age-adjusted models revealed a significant association between QOL and CKD, however this association no longer reached statistical significance after adjustment for confounders (table 2). In comparison, CKD was strongly associated with worse overall health in age-adjusted and multivariate-adjusted models. There was a significant trend of worsening kidney function across decreasing self-reported QOL and health status, however, this trend only remained significant in multivariate-adjusted analyses for health status (table 3).

We found no significant interactions between CKD and race for predicting any of the outcomes. We found an interaction of CKD and age for predicting depressive symptoms. In adjusted analyses, CKD was associated with a $0.37(95 \%$ CI $0.14-0.98)$ odds for depressive symptoms in those subjects $<60$ years, 0.88 ( $95 \%$ CI $0.40-1.94$ ) odds for depressive symptoms in those 60 to $<70$, and 1.31 (95\% CI $0.72-2.39)$ odds for those over 70 years ( $p$ for interaction $=0.01)$. Overall the prevalence of depressive symptoms decreased in each increasing age group, particularly in subjects without CKD. (fig. 1) We found no interactions between CKD and age for predicting overall health, QOL, or stress.

\section{Discussion}

After adjustment for age, subjects with CKD were more likely to have reduced QOL and poor overall health in the Heart and Soul Study compared with subjects with 
normal kidney function. Adjustment for confounders attenuated the association of CKD and QOL, however the relationship with health status remained significant. In contrast, we did not find an association of CKD with depressive symptoms, or perceived stress. Our findings suggest that mild to moderate CKD is associated with reduced self-assessed health and modestly decreased QOL, but that mental health outcomes do not appear to be affected by moderate reductions in renal function.

Our findings should be interpreted in the context of prior studies. In the MDRD Study (mean GFR $33 \mathrm{ml} /$ min), multivariate analyses showed a significant positive correlation between measured GFR and Quality of WellBeing score, a measure of health-related QOL [10]. However, in contrast to our study, a significant inverse relationship was found between measured GFR and the Symptom Checklist-90R scores, suggesting worsened mental health in subjects with reduced GFR. Shidler et al. [26] evaluated persons with moderate to severe kidney disease (mean creatinine clearance $37 \mathrm{ml} / \mathrm{min}$ ) and did not find depressive symptoms to be correlated to serum creatinine concentration. The AASK Trial (mean GFR $46 \mathrm{ml} / \mathrm{min}$ ) reported similar results to our study [13]; subjects in this cohort had lower physical component summary scores on the SF-36 than the general US population, however, the mental summary scores of the cohort were slightly higher than in the general population. A prior publication from the Heart and Soul Study found that CKD (creatinine clearance $<60 \mathrm{ml} / \mathrm{min}$ ) was associated with self-assessed physical limitation [11]. Thus, it appears that physical health declines at earlier stages of renal dysfunction than mental health.

Considering the physical symptoms that accompany $\mathrm{CKD}$, it is not surprising that we found an association of CKD with worsened overall health. However, it is interesting that moderate kidney disease appeared to be associated with decrements in overall health, and that mental health outcomes appeared similar in subjects with and without CKD. The association of CKD with QOL, although significant in age-adjusted analyses, was less robust than that of CKD with health status. It may be that subjects equate their overall health predominantly with physical health, while QOL encompasses a broader range of factors, including psychosocial outcomes. It is commonly accepted that QOL is reduced in persons with ESRD [2, 15] and depression is thought to be the most common psychological disorder in ESRD patients [27, 28], with estimated prevalence rates in excess of one-third $[9,29]$. However, the prevalence of depression in subjects with CKD has not been well studied [14]. Depression has been associated with the response to a loss of some kind [27] and stress is often a response to a change in a person's life [30]. Persons with advanced kidney disease have not only reduced physical function, but their loss in mobility may affect employment, familial roles, and social networks. These losses, coupled with the stress of dealing with the changes in the patients' lifestyle, may lead to the high prevalence of psychological illness in subjects with ESRD.

Mental health may remain unchanged in persons with moderate CKD because the burden of their illness has not yet grown to represent a change or loss. While these patients have a reduction in physical health, it may not substantially limit their performance in their daily life. Therefore, although physical health is affected, mental health outcomes appear to remain stable in the setting of moderate CKD. This may explain why we found a smaller reduction in overall QOL, compared with health status, because it reflects a combination of mental and physical health. Interestingly, the association of CKD with QOL appeared to be an average of the associations of CKD with overall health and the mental health outcomes.

We found an interesting interaction of age and CKD for predicting depressive symptoms. Although somewhat unexpected, previous studies have found an inverse association between age and depression, with depression being more prevalent in the young than in the elderly [ 31 , 32]. It is possible that despite declining physical health, such as coronary artery disease or CKD, the elderly adapt to their life situations more effectively. We also must consider the possibility that the interaction of age and CKD for predicting depressive symptoms may have been a chance finding.

A strength of our study is that, unlike most previous studies, we included persons with normal renal function as a comparison group. The data that exist regarding the association of health-related QOL and CKD are predominantly in cohorts with renal disease, and therefore do not include comparison groups without CKD. Another strength is the measurement of creatinine clearance by 24-hour urine collection, rather than a one-time measurement of serum creatinine.

Our study also has some limitations that must be considered. This was a cross-sectional analysis which limits our ability to determine causation. We cannot assume that CKD would be associated with the development of worsened health status over time. In addition, there may have been unmeasured confounding variables that may have affected the associations of CKD with our outcome variables. We cannot exclude the possibility that our pos- 
itive or negative findings may have been due to chance. The Heart and Soul Study was a cohort of predominantly male patients with coronary artery disease, therefore our results may not be applicable to women or to the general population. Finally, subjects were excluded from the study if they had a myocardial infarction in the past 6 months or were unable to walk one block; therefore our findings may not be generalizable to those with a recent coronary event or disability.

An additional potential limitation is the use of singleitem measures for both overall health and QOL. However, since our study aimed to compare the broad concepts of overall health and QOL between subjects with and without $\mathrm{CKD}$, we believe single-item measures were appropriate. Multiple-item measures may be more precise for specific treatment effects, but single-item measures are valid for overall comparisons between study populations [33]. A single-item measure of health has been shown to be predictive of mortality in elderly patients [34]. In addition, studies have shown single-item measures of health-related QOL to correlate positively and significantly with multiple-item instruments in persons with chronic disease $[24,33,35]$.
In conclusion, we found CKD to be independently associated with self-reported overall health, and modestly associated with QOL in a cohort of persons with coronary artery disease. However, moderate CKD was not associated with depressive symptoms, or perceived stress. These findings suggest that although overall health is diminished in subjects with moderate kidney disease, mental health may remain unchanged, resulting in only a modest reduction in overall QOL. Longitudinal studies of renal function with overall health, mental health, and QOL outcomes will help us to understand their relation over time.

\section{Acknowledgments}

The Heart and Soul Study was funded by grants from the Department of Veterans Affairs (Epidemiology Merit Review Program), the Robert Wood Johnson Foundation (Generalist Physician Faculty Scholars Program), the American Federation for Aging Research (Paul Beeson Faculty Scholars in Aging Research Program), the Ischemia Research and Education Foundation, and the University of California, San Francisco (Hellman Family Award). None of the funding sources had any role in the collection of data, interpretation of results, or preparation of the manuscript.

\section{References}

1 Blake C, Codd MB, Cassidy A, O'Meara YM: Physical function, employment and quality of life in end-stage renal disease. J Nephrol 2000; 13:142-149.

-2 Evans RW, Manninen DL, Garrison LP Jr, Hart LG, Blagg CR, Gutman RA, Hull AR, Lowrie EG: The quality of life of patients with end-stage renal disease. N Engl J Med 1985; 312:553-559.

- 3 DeOreo PB: Hemodialysis patient-assessed functional health status predicts continued survival, hospitalization, and dialysis-attendance compliance. Am J Kidney Dis 1997;30:204212.

4 Painter P, Carlson L, Carey S, Paul SM, Myll $\mathrm{J}$ : Physical functioning and health-related quality-of-life changes with exercise training in hemodialysis patients. Am J Kidney Dis 2000; 35:482-492.

-5 Kimmel PL, Peterson RA, Weihs KL, Simmens SJ, Alleyne S, Cruz I, Veis JH: Multiple measurements of depression predict mortality in a longitudinal study of chronic hemodialysis outpatients. Kidney Int 2000;57:2093-2098.

-6 Craven JL, Rodin GM, Littlefield C: The Beck Depression Inventory as a screening device for major depression in renal dialysis patients. Int J Psychiatry Med 1988;18:365-374.

7 Hinrichsen GA, Lieberman JA, Pollack S, Steinberg H: Depression in hemodialysis patients. Psychosomatics 1989;30:284-289.
Smith MD, Hong BA, Robson AM: Diagnosis of depression in patients with end-stage renal disease. Comparative analysis. Am J Med 1985;79:160-166.

-9 Walters BA, Hays RD, Spritzer KL, Fridman M, Carter WB: Health-related quality of life, depressive symptoms, anemia, and malnutrition at hemodialysis initiation. Am J Kidney Dis 2002; 40:1185-1194.

10 Rocco MV, Gassman JJ, Wang SR, Kaplan RM: Cross-sectional study of quality of life and symptoms in chronic renal disease patients: the Modification of Diet in Renal Disease Study. Am J Kidney Dis 1997;29:888-896.

11 Odden MC, Whooley MA, Shlipak MG: Association of chronic kidney disease and anemia with physical capacity: the Heart and Soul Study. J Am Soc Nephrol 2004;15:29082915.

12 Shlipak MG, Stehman-Breen C, Fried LF, Song X, Siscovick D, Fried LP, Psaty BM, Newman AB: The presence of frailty in elderly persons with chronic renal insufficiency. Am J Kidney Dis 2004;43:861-867.

13 Kusek JW, Greene P, Wang SR, Beck G, West D, Jamerson K, Agodoa LY, Faulkner M, Level B: Cross-sectional study of health-related quality of life in African Americans with chronic renal insufficiency: the African-American Study of Kidney Disease and Hypertension Trial. Am J Kidney Dis 2002;39:513-524.
14 Kimmel PL: Depression in patients with chronic renal disease: what we know and what we need to know. J Psychosom Res 2002;53: 951-956.

15 Valderrabano F, Jofre R, Lopez-Gomez JM: Quality of life in end-stage renal disease patients. Am J Kidney Dis 2001;38:443-464.

16 Ruo B, Rumsfeld JS, Hlatky MA, Liu H, Browner WS, Whooley MA: Depressive symptoms and health-related quality of life: the Heart and Soul Study. JAMA 2003;290:215-221.

17 Beattie MS, Shlipak MG, Liu H, Browner WS, Schiller NB, Whooley MA: C-reactive protein and ischemia in users and nonusers of $\beta$-blockers and statins: data from the Heart and Soul Study. Circulation 2003; 107:245-250.

18 National Kidney Foundation NKF-K/DOQI clinical practice guidelines for anemia of chronic kidney disease: update 2000. Am J Kidney Dis 2001;37:S182-S238.

19 Ix JH, Shlipak MG, Liu HH, Schiller NB, Whooley MA: Association between renal insufficiency and inducible ischemia in patients with coronary artery disease: the Heart and Soul Study. J Am Soc Nephrol 2003; 14:32333238.

20 Odden MC, Whooley MA, Shlipak MG: Association of chronic kidney disease and anemia with physical capacity: the Heart and Soul Study. J Am Soc Nephrol 2004;11:29082915. 
21 Spitzer RL, Kroenke K, Williams JB: Validation and utility of a self-report version of PRIME-MD: the PHQ primary care study. Primary care evaluation of mental disorders. Patient Health Questionnaire. JAMA 1999; 282:1737-1744.

-22 Kroenke K, Spitzer RL, Williams JB: The PHQ-9: validity of a brief depression severity measure. J Gen Intern Med 2001;16:606613.

-23 Cohen S, Kamarck T, Mermelstein R: A global measure of perceived stress. J Health Soc Behav 1983;24:385-396.

24 Cunny KA, Perri M 3rd: Single-item vs. multiple-item measures of health-related quality of life. Psychol Rep 1991;69:127-130.

25 Guyatt G, Feeny D, Patrick D: Issues in quality-of-life measurement in clinical trials. Control Clin Trials 1991;12:S81-S90.
6 Shidler NR, Peterson RA, Kimmel PL: Quality of life and psychosocial relationships in patients with chronic renal insufficiency. Am J Kidney Dis 1998;32:557-566.

27 Kimmel PL, Weihs K, Peterson RA: Survival in hemodialysis patients: the role of depression. J Am Soc Nephrol 1993;4:12-27.

28 Kimmel PL: Psychosocial factors in adult endstage renal disease patients treated with hemodialysis: correlates and outcomes. Am J Kidney Dis 2000;35:S132-140.

29 Finkelstein FO, Finkelstein SH: Depression in chronic dialysis patients: assessment and treatment. Nephrol Dial Transplant 2000; 15: 1911-1913.

30 McEwen BS, Seeman T: Protective and damaging effects of mediators of stress. Elaborating and testing the concepts of allostasis and allostatic load. Ann NY Acad Sci 1999;896:3047.

31 Weissman MM, Bruce ML, Leaf PJ, Florio LP, Holzer C: Psychiatric disorders in America: the epidemiologic catchment area study; in Robins LE, Regier DA (eds): Affective Disorders. New York, Free Press, 1991.
32 Gottlieb SS, Khatta M, Friedmann E, Einbinder L, Katzen S, Baker B, Marshall J, Minshall S, Robinson S, Fisher ML, Potenza M, Sigler B, Baldwin C, Thomas SA: The influence of age, gender, and race on the prevalence of depression in heart failure patients. J Am Coll Cardiol 2004;43:1542-1549.

33 Bernhard J, Sullivan M, Hurny C, Coates AS, Rudenstam CM: Clinical relevance of singleitem quality of life indicators in cancer clinical trials. Br J Cancer 2001;84:1156-1165.

34 Mossey JM, Shapiro E: Self-rated health: a predictor of mortality among the elderly. Am J Public Health 1982;72:800-808.

35 Deniston OL, Carpentier-Alting P, Kneisley J, Hawthorne VM, Port FK: Assessment of quality of life in end-stage renal disease. Health Serv Res 1989;24:555-578. 